\title{
Design of Spring Coupling for High- $Q$ High-Frequency MEMS Filters for Wireless Applications
}

\author{
Mohammed M. Shalaby, Mohamed A. Abdelmoneum, Member, IEEE, and Kazuhiro Saitou, Senior Member, IEEE
}

\begin{abstract}
A second-order microelectromechanical systems (MEMS) filter with high selectivity and sharp rolloff is required in wireless transceivers used in dense wireless sensor networks (WSNs). These sensors are expected to replace existing wired sensors used in industrial-plant management and environmental monitoring. These filters, together with MEMS-based oscillators and mixers, are expected to replace off-chip components and enable the development of a single-chip transceiver. Such a transceiver will leverage the integrated MEMS components' characteristics to operate at lower power and, hence, longer battery life, making autonomous WSNs more feasible in a wider range of applications. As a result, this paper presents the design and optimization of the coupling beam of wineglass-mode micromechanical disk filters using simulated annealing. The filter under consideration consists of two identical wineglass-mode disk resonators, mechanically coupled by a flexural-mode beam. The coupled two-resonator system exhibits two mechanical-resonance modes with closely spaced frequencies that define the filter passband. A constraint is added on the beam length to eliminate the effect of the coupling-beam mass on the filter's resonant frequency. A new process flow is proposed to realize self-aligned overhanging coupling beams designed in this paper.
\end{abstract}

Index Terms-High-frequency microelectromechanical systems (MEMS), micromechanical filters, optimization, simulated annealing, spring coupling.

\section{NOMENCLATURE}

$f_{o} \quad$ Resonance frequency.

$R \quad$ Disk radius.

$\rho \quad$ Material density.

$\nu \quad$ Poisson's ratio.

$E \quad$ Young's modulus of elasticity.

$\omega_{0}=2 \pi f_{o} \quad$ Natural angular frequency.

$U \quad$ Radial-displacement component.

$V \quad$ Tangential-displacement component.

$m_{\text {re }} \quad$ Resonator effective mass.

$K_{\text {re }} \quad$ Resonator effective stiffness.

Manuscript received April 29, 2008; revised January 12, 2009. First published February 6, 2009; current version published April 1, 2009.

M. Shalaby was with the University of Michigan, Ann Arbor, MI 48109 USA. He is now with the General Electric Global Research Center, Niskayuna, NY 12309 USA (e-mail: mshalaby@umich.edu).

M. Abdelmoneum was with the University of Michigan, Ann Arbor, MI 48109 USA. He is now with Intel Corporation, Hillsboro, OR 97124-6497 USA (e-mail: mohamed.a.abdel-moneum@intel.com).

K. Saitou is with the Department of Mechanical Engineering, University of Michigan, Ann Arbor, MI 48109 USA (e-mail: kazu@ umich.edu).

Color versions of one or more of the figures in this paper are available online at http://ieeexplore.iee.org.

Digital Object Identifier 10.1109/TIE.2009.2014671

\section{INTRODUCTION}

W IRELESS sensor networks (WSNs) are expected to dominate industrial and environmental sensing networks to replace the current wired architecture not only to reduce cost but also to increase flexibility, scalability, and interoperability [1], [2]. Cost overhead stems from the wiring and maintenance costs associated with wired technologies [2], [3]. To reduce such cost, a low-cost wireless transceiver has to be integrated with the sensor to enable data transmission to the main command and control unit. The cost of a wireless transceiver can be significantly reduced if it can be manufactured using a batch process. This will eliminate the use of printed circuit boards used to integrate different subsystems [4]; thus, reduce cost. Such integration covers all off-chip passive components such as antennas [5], capacitors [6], inductors [7], and frequency selection and generation blocks [8].

In addition, new domains that were very hard to monitor with wired technologies can now be exploited using wireless sensors. For example, wireless sensors would be the only practical option for high-resolution crops monitoring where wired sensors cannot be used. Wireless sensors can enable monitoring harsh environments where environmental constraints and available space for sensor limits the use of wired networks. This is a typical scenario in chemical plants, semiconductor fabrication plants, power plants, and environmental monitoring [9]. In any of these wireless-sensor applications, size, power, cost, and performance of the wireless transceiver play an important role in enabling the WSNs.

Frequency selecting and generating components, namely, filters and oscillators, are inherent building blocks in any modern wireless-transceiver system as they play a key role in determining the overall system performance and sensitivity [10]-[13]. In current systems, off-chip mechanically resonant components, such as crystal resonators and filters [14], surfaceacoustic-wave (SAW) filters [15], [16], and film bulk acoustic resonators [17], [18], are currently used to realize high- $Q$ bandpass filters, commonly used in the radio-frequency (RF) and intermediate-frequency (IF) stages of heterodyne transceivers [12], [13]. These mechanical components have higher quality factors as compared to their transistor-based counterparts [3], [5]; as a result, they greatly outperform comparable filters implemented using transistor technologies [13], [15]. On the other hand, these mechanical devices are bulky and cannot be integrated on-chip owing to their non-CMOS-compatible fabrication technology; as a result, the overall system cost increases. 
In addition, the insertion loss associated with these off-chip components affects the system performance and reduces the battery lifetime if additional amplification stages are required [8], [11]. For these reasons, research on how to implement microelectromechanical-systems (MEMS)-based filters and oscillators is currently an active area of research [8], [19], [20].

The remainder of this paper is organized as follows: Section II presents earlier work done in this field. Then, a description of the micromechanical-filter structure and operation is presented in Section III. The theory behind the highfrequency (HF) micromechanical-resonator and filter design are presented in Sections IV and V, respectively. Section VI presents the optimization of the coupling-beam dimensions and the coupling locations using simulated annealing. Finally, Section VII describes the fabrication process for realizing the designed filter.

\section{RELATED WORK}

Advances in surface-micromachining technologies made it possible to fabricate on-chip high- $Q$ micromechanical resonators (abbreviated as " $\mu$ resonators") and filters [21], [22]. Different approaches have been recently applied to tackle various performance aspects of MEMS resonators. These approaches were successful in positioning vibrating RF MEMS technology as a serious option to replace off-chip counterparts. Namely, low-cost wafer-level vacuum packaging [23], CMOS integration [24], [25], temperature compensation [26], and higher frequency operation [8] would enable low-cost on-chip RF MEMS resonators that can eventually outperform existing technologies.

Vibrating MEMS wineglass-mode disk resonators fabricated using polysilicon as the structural material, first demonstrated by Abdelmoneum et al. [27], [28], exhibit $Q$ 's of more than 96000 under vacuum and 8600 in atmosphere and center frequency around $71 \mathrm{MHz}$ [27] and, later, with $Q$ 's in the excess of 145000 in vacuum [29]. These resonators were incorporated in demonstrating reference oscillators with phase-noise performance surpassing Global System for Mobile communications requirements [8]. At this point, it appears that $\mu$ resonators can potentially serve well as miniaturized substitutes for crystals in a variety of high- $Q$ oscillator and filtering applications.

IF (i.e., $455 \mathrm{kHz}$ ) MEMS filters were demonstrated early in literature [30]; later, higher frequency MEMS filters with frequencies around $7.8 \mathrm{MHz}$ and percent bandwidths from $0.2 \%$ to $2.5 \%$ ( $Q$ from 40 to 450 ) were also demonstrated [31]. Unfortunately, filters working at much higher frequencies are still needed for useful implementation in communication systems, in particular at RF range [11], [32]. This paper focuses on frequencies in the vicinity of $71 \mathrm{MHz}$ and percent bandwidth from $0.14 \%$ to $1.6 \%$ ( $Q$ from 62 to 710 ); however, practical implementation is not limited to this range.

\section{Filter Structure and Operation}

The filter consists of two identical mechanical wineglassmode disk resonators, mechanically coupled by a flexural-mode or an extensional-mode beam, all suspended at $4000 \AA$ above

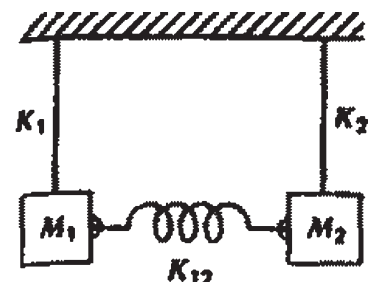

(a)

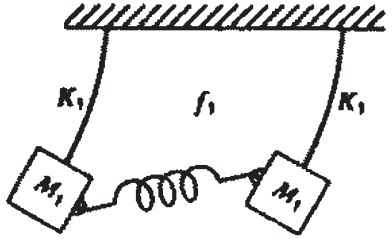

(c)

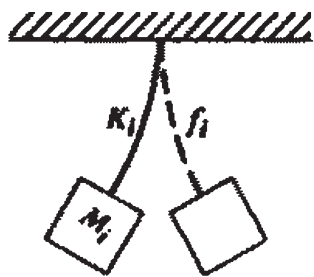

(b)

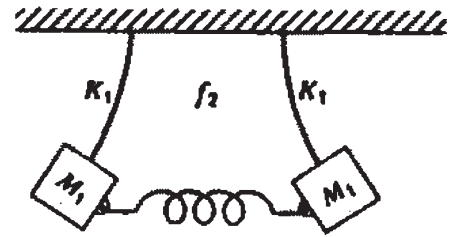

(d)
Fig. 1. Coupled spring-mass resonators. (a) Resonators at rest. (b) Single resonator natural mode. (c) In-phase mode. (d) Out-of-phase mode [33].

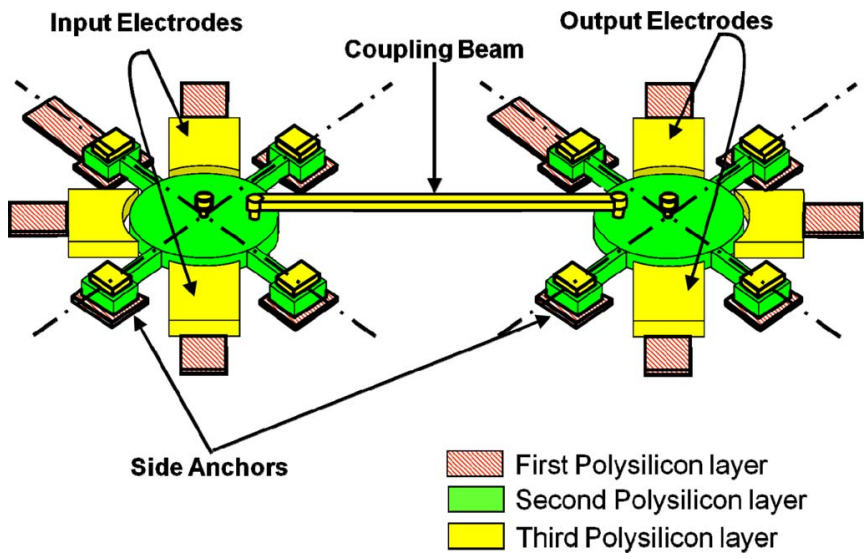

Fig. 2. Schematic for the wineglass-mode filter fabricated using the selfaligned process.

the substrate. A capacitive transducer electrode is positioned on the side of one of the resonators to excite the resonator with the desired wineglass vibration mode. Another electrode is positioned on the side of the other resonator to read the output response from the filter.

Such a coupled two-resonator system exhibits two mechanical-resonance modes with closely spaced frequencies that define the filter passband. Fig. 1 shows the equivalent lumped-mass mechanical system of the filter. The frequencies of the constituent resonators determine the center frequency of the filter, while the bandwidth is determined by the stiffness of the coupling spring and the coupling location. Using the earlier procedure, wineglass resonators can be mechanically coupled as shown in Fig. 2. As shown in Fig. 4, each mode peak corresponds to a distinct physical-mode shape. In the lower frequency mode, the resonators are $180^{\circ}$ out of phase, and in the higher frequency mode, both resonators vibrate in phase.

The operation of this filter can be seen as a device that takes an electrical input signal, converts it to mechanical signal, processes this signal in the mechanical domain, then converts the resulting signal once more to an electrical output 


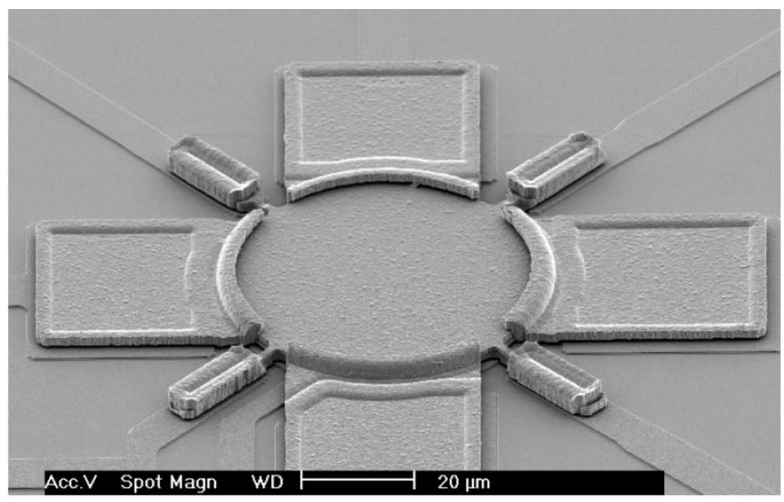

(a)

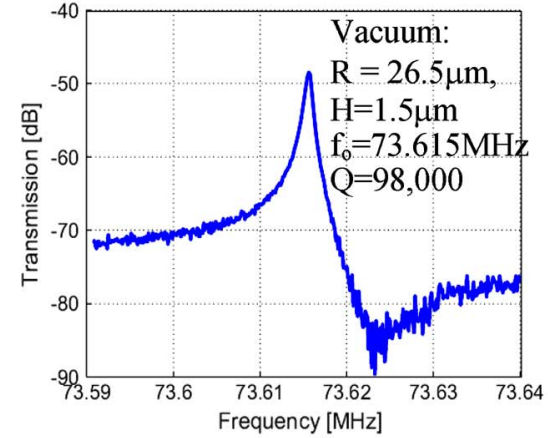

(b)

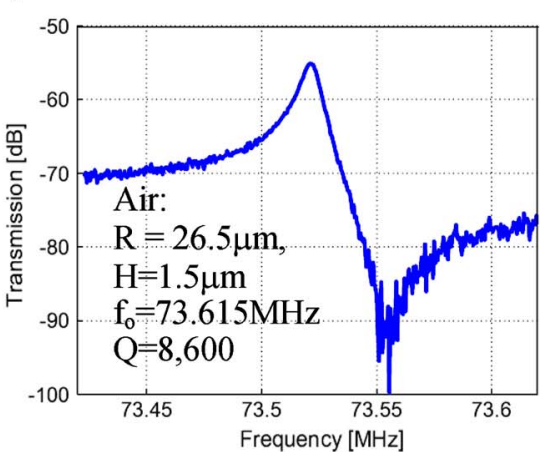

(c)

Fig. 3. Wineglass-mode resonator. (a) SEM picture. Measured frequency spectrum (b) in vacuum and (c) in air [27].

signal, ready for further processing by subsequent electronic stages.

\section{HF $\mu$ RESONATOR}

HF stemless wineglass-mode disk resonator has been presented recently by Abdelmoneum et al. [27]. Fig. 3(a) shows the SEM of the wineglass-mode disk resonator. The resonator features four anchored supports attached to the disk at its four wineglass nodal points. This wineglass resonator is able to operate with substantially high $Q$.

The electrodes are located around the circumference of the disk, one in each of the four quadrants about $1000 \AA$ from the disk and are used for electrostatic excitation and detection. To select the wineglass-mode shape, identical signals should be applied on opposing electrodes along one axis. The sensing can be done using the electrodes on the orthogonal axis; they can also be connected to an out-of-phase input signal to add additional drive force.

Fig. 3(b) and (c) shows the measured frequency response for a $73-\mathrm{MHz}$ version measured in vacuum and in air, respectively. With only two successive perimeter nodal supports, $Q$ was about 98000 in vacuum and 8600 in air. The reasons of such high $Q$ are as follows: 1) unlike clamped-beam resonators, the disk supports are at the four nodal points where the displacement is almost zero, so minimum energy is transferred to substrate through anchors; 2) fabrication error in support location is minimum, because they are done in the same stage the resonator is deposited, unlike stemmed wineglass resonators; and 3) squeeze-film damping effect for this resonator is minimal, since the resonator vibrates in plane, the surface area is very small as compared to that of plane clamped-beam resonators.

\section{A. Resonator Governing Equation}

The wineglass-mode disk vibrates in an elliptical-mode shape as shown in Fig. 4. This mode is a compound mode in which the displacement vector consists of two orthogonal components, a tangential component, and a radial one. Assuming that the disk thickness is much smaller than its radius, the disk can be approximated as a thin circular plate. The mode equation for the lowest order compound mode for a circular plate was derived by Onoe [34], where the mode frequency $f_{o}$ for a wineglass-mode disk can be obtained by solving the mode frequency equation [34]

$$
\left[\Psi_{2}\left(\frac{\zeta}{\xi}\right)-2-q\right]\left[2 \Psi_{2}(\zeta)-2-q\right]=(n q-n)^{2}
$$

where

$$
q=\frac{\zeta^{2}}{2 n^{2}-2} \quad \zeta=R \sqrt{\frac{2 \rho \omega_{o}^{2}(1+\nu)}{E}} \quad \xi=\sqrt{\frac{2}{1-\nu}} .
$$

$R$ is the disk radius, $\omega_{o}=2 \pi f_{o}$ is the angular frequency, $n=2$ is the mode order, and $\rho, \nu$, and $E$ are the density, Poisson ratio, and Young's modulus, respectively, of the disk structural material.

The effective mass of resonator at any point on the disk periphery can be evaluated from

$$
\frac{1}{2} m_{\mathrm{re}} v_{\mathrm{re}}^{2}=K E_{\text {total }}
$$

where $K E_{\text {total }}$ is the total kinetic energy of the resonator and is obtained by integrating over the resonator volume. $K E_{\text {total }}$ is equal to the summation (integration) of the product of an infinitesimal mass and the total velocity of that mass. The computed 


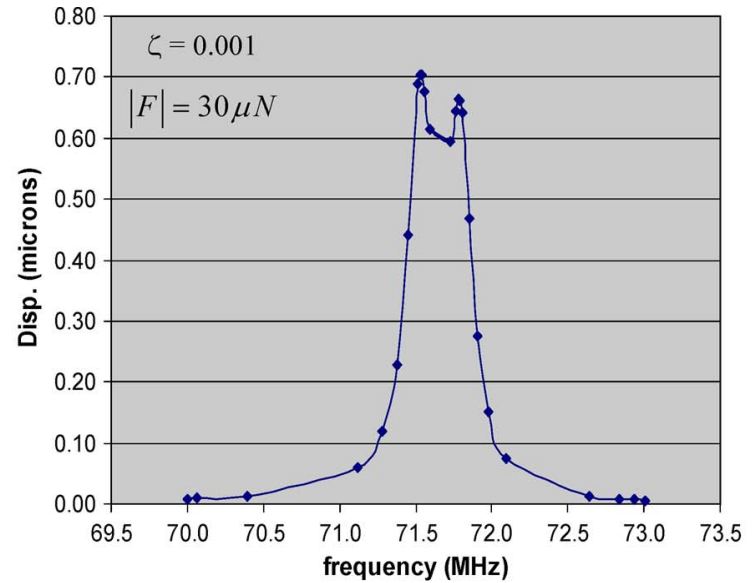

(a)

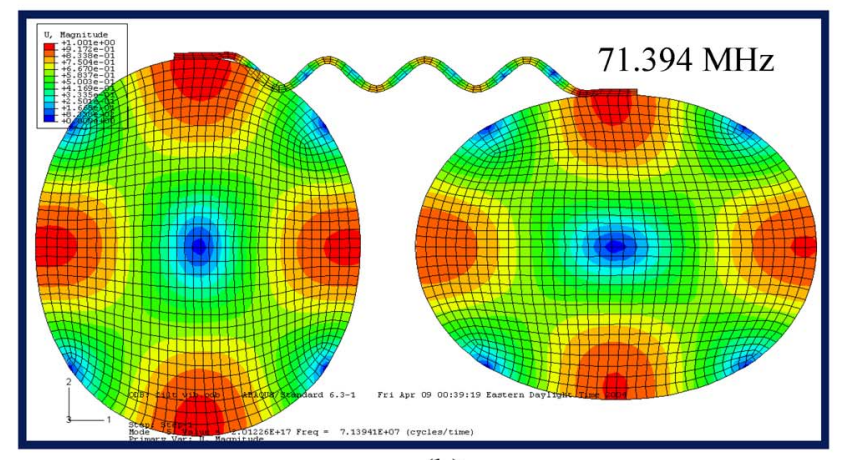

(b)

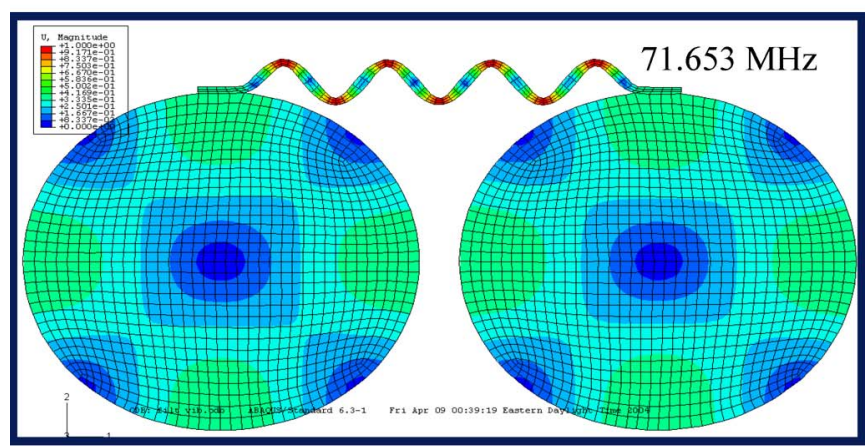

(c)

Fig. 4. Simulation for filter flexural-mode coupling. (a) Unterminated frequency-response plot. (b) Out-of-phase mode. (c) In-phase mode.

total kinetic energy is also equal to the product of the effective mass at a specific location $m_{\mathrm{re}}$ and the corresponding velocity $v_{\text {re }}$ at the same location. Implementing the wineglass-mode disk-resonator equation, the earlier equation can be rewritten as given in

$m_{\mathrm{re}}=\frac{\rho \pi H \int_{0}^{R} r\left[\frac{\zeta}{2 R \xi} J_{1}\left(\frac{\zeta r}{\xi R}\right)-\frac{\zeta}{2 R \xi} J_{3}\left(\frac{\zeta r}{\xi R}\right)+\frac{B}{r A} J_{2}\left(\frac{\zeta r}{R}\right)\right]^{2} d r}{\left[\frac{\zeta}{2 R \xi} J_{1}\left(\frac{\zeta r_{c}}{\xi R}\right)-\frac{\zeta}{2 R \xi} J_{3}\left(\frac{\zeta r_{c}}{\xi R}\right)+\frac{B}{r_{c} A} J_{2}\left(\frac{\zeta r_{c}}{R}\right)\right]^{2}}$

where $H$ is the disk thickness, $B / A=-4.5236$, and $J_{n}$ is Bessel function of the first type and order $n$. Now, the effective stiffness at the coupling location can be evaluated using

$$
K_{\mathrm{re}}=\omega^{2} m_{\mathrm{re}} .
$$

\section{Micromechanical-Filter Design}

The filter design is dominantly governed by the bandwidth equation

$$
\mathrm{BW}=\frac{f_{o}}{K_{i j}} \frac{K_{c}}{K_{\mathrm{re}}}
$$

where $f_{o}$ is the center frequency of the filter, it is also the resonant frequency of each of the resonators, $K_{i j}$ is the normalized coupling coefficient ( $K_{i j}=0.7225$ [35]), $K_{c}$ is the couplingbeam stiffness, and $K_{r}$ is the resonator effective stiffness at the coupling location. It is obvious that the bandwidth depends only on the resonator stiffness, i.e., the coupling location on the resonators, and the coupling-beam stiffness. The other two parameters are constants. In such microscale filters, it may not be possible to design a highly compliant coupling beam for desired low-bandwidth filters. As a result, the coupling location plays an important role in determining the filter bandwidth as well, as it affects the resonator effective stiffness. As will be shown later, if the coupling is at low-velocity location on the resonator, the effective mass increases and so is the effective stiffness, consequently, the filter bandwidth decreases.

\section{A. Coupling-Beam Governing Equations}

Extensional- and torsional-mode coupling elements have similar equations. The stiffness of the coupling beam is given by

$$
K_{c}=\omega A \sqrt{\rho E} \quad l=\frac{n \lambda}{4}, \quad n=1,3,5, \ldots
$$

In this microscale, the mass of the coupling beam and that of the resonator are of the same order. As a result, it is important to account for the coupling-beam mass. Since this is not an easy process, an easier way is to set the beam length equal to an odd multiple of quarter the wavelength. By doing so, the couplingbeam mass should have no effect on the resonator frequency [30], [36].

For the flexural-mode coupling with clamped ends, the beam stiffness is calculated using

$$
K_{c}=\frac{E I \alpha^{3}(\sin \alpha+\sinh \alpha)}{L^{3}(\cos \alpha \cosh \alpha-1)}, \quad \alpha=\sqrt[4]{\frac{\rho A \omega^{2}}{E I}} L .
$$

Again, it is important to find the coupling-beam dimensions that correspond for the quarter-wavelength to eliminate the effect of the coupling-beam mass. As a result, the equality given by (9) should be satisfied

$$
H 6=\sin \alpha \cosh \alpha+\cos \alpha \sinh \alpha=0 .
$$

\section{B. Simulations for Extensional-Mode Filters}

A coupling-beam width of $1 \mu \mathrm{m}$ was chosen; beam stiffness and beam length were obtained from (7). The coupling location was chosen to be at the outer radius of the resonators. Using (3), the BW is $1.14 \mathrm{MHz}$. Fig. 5(a) and (b) shows the filter's simulated peak frequencies, while Fig. 5(c) shows the filter's unterminated frequency-response plot. 


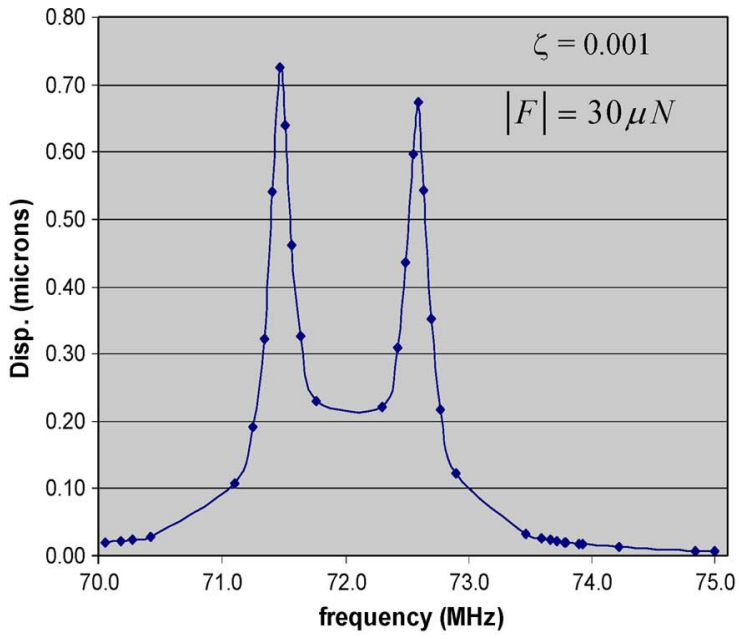

(a)

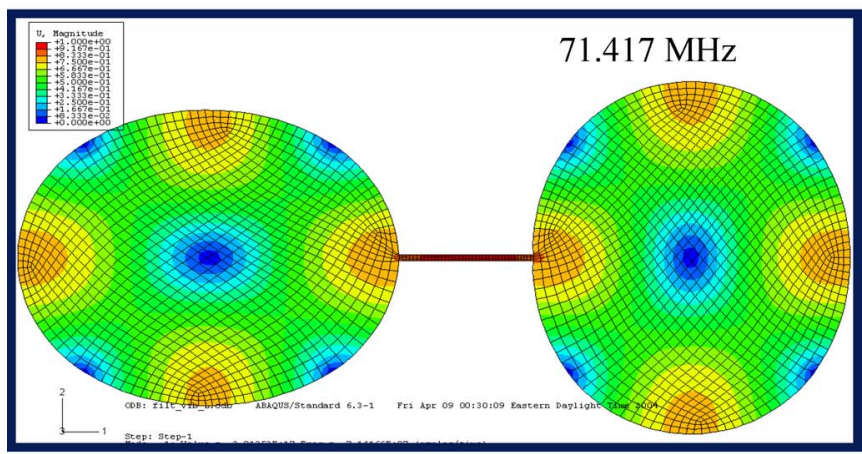

(b)

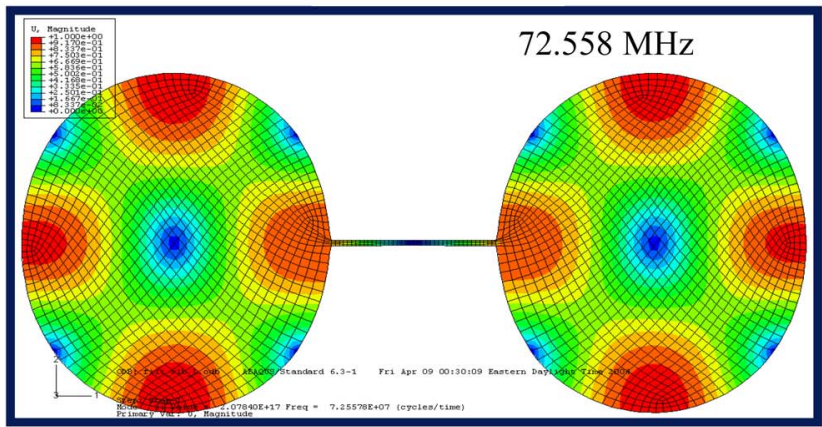

(c)

Fig. 5. Simulation for filter with extensional-mode coupling. (a) Unterminated frequency-response plot. (b) Out-of-phase mode. (c) In-phase mode.

\section{Simulations for Flexural-Mode Filters}

The coupling-beam width was chosen equal to $1 \mu \mathrm{m}$ (limited by the lithography resolution available in the university fabrication plant) and beam stiffness and beam length were obtained from (8). The coupling location was chosen to be at the outer radius of the resonators. Using (3), the bandwidth was $259 \mathrm{kHz}$. Fig. 4 shows the filter's simulated peak frequencies as well as the unterminated frequency-response plot.

\section{FILTER OPtimizATION FOR A DESIRED BANDWIDTH}

It is clear that the bandwidth for the extensional mode coupling is much higher than that for the flexural-mode coupling. This is due to the fact that the beam stiffness in extensional mode is much higher than its stiffness in flexural mode. In conclusion, if the desired bandwidth is in the range of hundreds of kilohertz, flexural-mode coupling beams should be used. While for bandwidths on the order of megahertz, extensionalmode coupling beams should be used.

Since filters are designed to have a certain bandwidth at a certain given frequency, the objective in this section is to find a wineglass-mode filter with a bandwidth of $100 \mathrm{kHz}$. Optimization is used to reach the desired target bandwidth.

\section{A. Definition of the Design Variables}

It can be deduced from (6) that the bandwidth depends on the resonator stiffness and the coupling-beam stiffness. Equations (4) and (5) show that the resonator stiffness can be controlled by controlling the resonator thickness $H$ and the coupling location $r_{c}$, while, from (7) and (8), the coupling-beam stiffness is controlled by the coupling-beam dimensions (length, width, and thickness).

Since the thicknesses of the beam and the resonator are controlled by the manufacturing process, they are kept fixed during optimization. Thus, the selected design variables are the beam length $(l)$, the beamwidth $(w)$, and the coupling location from the center $\left(r_{c}\right)$.

\section{B. Definition of the Constraints}

The upper and lower bounds of the beamwidth are again governed by the manufacturing process. The coupling location has to be off the disk center and along the vertical direction to transfer all the vertical energy without any horizontal energy component. Thus, the beam length has to be at least equal to the disk diameter. In addition, if the beam length exceeds a certain value, (7) and (8) become unsolvable because of their extreme nonlinearity. Using the earlier two conditions, the upper and lower bounds of the beam length are obtained. The upper bound over the coupling location is simply the beam radius. In addition, two more equality constraints are needed to ensure that the resulting bandwidth is equal to $100 \mathrm{kHz}$ and that (9) is satisfied. Mathematically, these constraints can be written as

$$
\begin{aligned}
0.75 & \leq w \leq 2.0 \mu \mathrm{m} \\
55 & \leq l \leq 90 \mu \mathrm{m} \\
10 & \leq r_{c} \leq 26.5 \mu \mathrm{m} \\
h_{1} & =a b s\left(\mathrm{BW}-100 \times 10^{3}\right)=0 \\
h_{2} & =H 6=0 .
\end{aligned}
$$

\section{Definition of the Design Objective}

Since the objective here is to reach a certain bandwidth, the objective can be easily handled as a constraint, as shown earlier. In addition, since the satisfaction of the second equality constraint $\left(h_{2}\right)$ is too hard due to the high nonlinearity of the equation, the authors considered minimizing the absolute value of $\mathrm{H} 6$ as the design objective instead, as shown in (15). This way, $H 6$ can get as close as possible to zero, as needed, without really affecting the actual problem. As a result, $h_{2}$ is no longer 


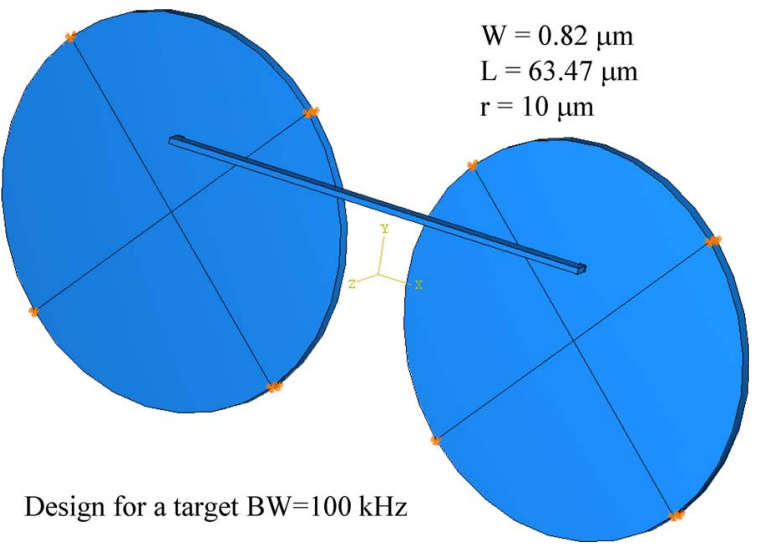

Fig. 6. Three-dimensional model of the proposed 100-kHz bandwidth filter.

considered as a constraint and is removed from constraints equations, given earlier

$$
\min (a b s(H 6)) .
$$

\section{Optimization Method}

Simulated annealing [37], [38], first introduced by Kirkpatrick et al. [37], is a global stochastic optimization method capable of finding optimal or near-optimal solutions to problems with ill-behaved objective functions which resist optimization by traditional gradient-based techniques. Simulated annealing has a broad range of use in realistic engineering problems because they are often characterized by poorly behaved objective functions [39].

The high nonlinearity in the objective function and the constraints, in the current optimization problem, prevented normal-gradient-based algorithms, such as sequential quadratic programming [40] from performing well. As a result, simulated annealing was used, and it was able to reach a near-optimal solution (the detailed algorithm is presented in [41]).

The optimum values of the design variables are $l=$ $63.47 \mu \mathrm{m}, w=0.82 \mu \mathrm{m}$, and $r_{c}=10 \mu \mathrm{m}$. A 3-D model for the filter is shown in Fig. 6. Fig. 7(b) and (c) shows the filter's simulated peak frequencies, while Fig. 7(a) shows the filter's unterminated frequency-response plot.

\section{FABRICATION AND LAYOUT}

The first demonstrated wineglass-mode disk resonator was fabricated using a self-aligned surface-micromachining process that uses polysilicon as the structural material [27]. This selfaligned process eliminates $Q$ degradation resulting from anchor losses that take place due to anchor misalignment (during the anchor-patterning step) to the mode nodal points [42]. The selection of the disk structural material is not limited to polysilicon only; higher frequencies can be achieved using materials with higher modulus of elasticity like diamond and silicon carbide [28]. The proposed filter can be fabricated using the same self-aligned processes used to fabricate the first wineglass-mode resonator [27]. Fig. 2 shows a 3-D schematic of the wineglass-mode disk filter using the concept of the wineglass-mode disk resonator, first demonstrated in [27]. The

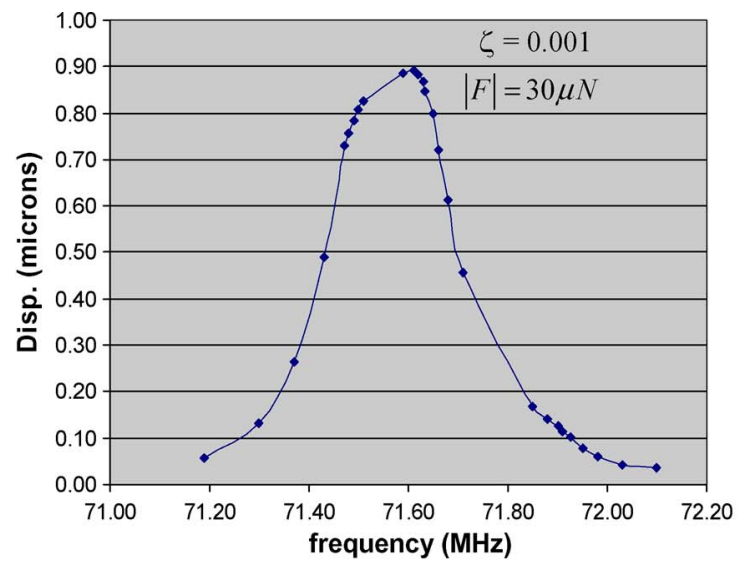

(a)

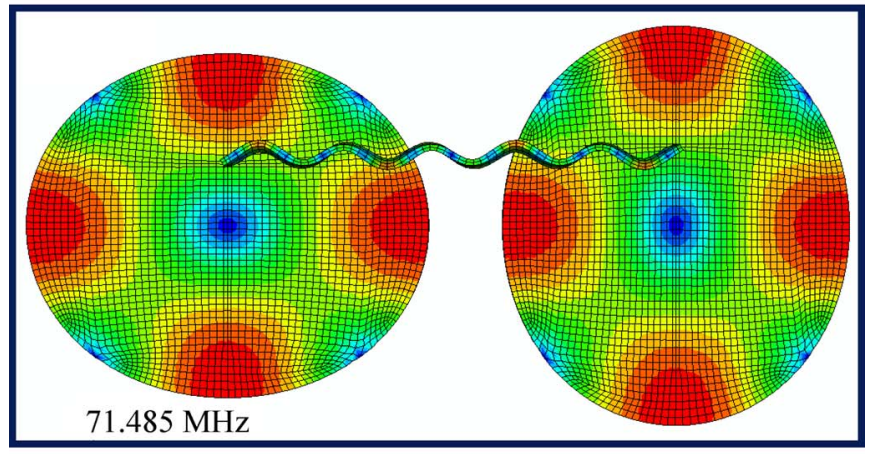

(b)

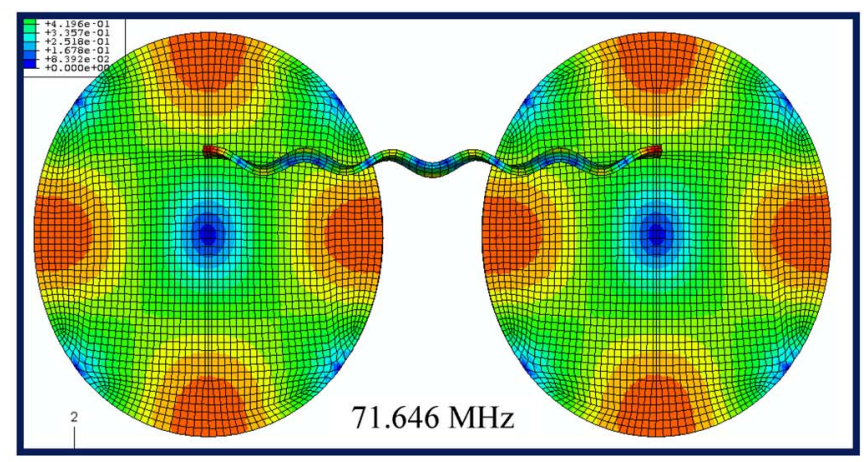

(c)

Fig. 7. Simulation for filter with $100-\mathrm{kHz}$ bandwidth. (a) Out-of-phase mode. (b) In-phase mode. (c) Unterminated frequency-response plot.

device consists of three polysilicon layers. The first layer is a 3000-Å-thick layer that defines the input/output and bias interconnects. The second layer is a $2-\mu \mathrm{m}$ structural layer that simultaneously defines the disk structure and anchor openings (self-aligned layer). The third layer is a $2-\mu \mathrm{m}$ layer that refills the anchor openings and make contact to the interconnect layer and defines the input/output electrodes. Figs. 8-15 show each of the aforementioned steps.

The process starts by forming a passivation layer on top of a bare silicon substrate. The passivation layer is composed of a 2- $\mu \mathrm{m}$-thick oxide layer followed by the deposition of $0.3-\mu \mathrm{m}$ silicon nitride layer, both deposited in the same furnace using low-pressure chemical vapor deposition (LPCVD). Following the deposition of the passivation layers, a 3000-Å polysilicon layer is then deposited using LPCVD, doped using phosphorus 
oxychloride (POCL) doping, and patterned using reactive-ion etching (RIE). This silicon layer serves as the "interconnects" layer (first polysilicon layer shown in Fig. 2) which provides input/output/bias lines to the device. To isolate the structural polysilicon layer (second polylayer shown in Fig. 2) to be deposited later from the formed interconnect polylayer, a $0.5-\mu \mathrm{m}$ oxide layer is deposited using LPCVD. A 2- $\mu \mathrm{m}$ structural polylayer is then deposited using LPCVD, doped using POCL doping, and then followed by depositing a $0.5-\mu \mathrm{m}$ oxide hardmask layer. The top oxide hard mask is then patterned to form the mask for the disk structure, coupling-beam opening, and center-stem anchor opening. This pattern is then transferred to the underlying structural polylayer using RIE. This is the selfaligned step in which both of the disk structure, coupling-beam opening, and the anchors are defined in the same patterning step, thus eliminating any misalignment between the anchor and the mode nodal points. The capacitive gap separating the disk structure from the electrodes (to be deposited and patterned) is then defined by depositing $1000 \AA$ of oxide. This thin oxide layer will be etched away during the release step to form the capacitive air gap. It should be noted that other high dielectric materials (high $K$ ) can be deposited instead of oxide.

The use of high- $K$ materials to form the capacitive gap is interesting as it can substantially reduce the motional resistance of the resonator and, hence, alleviate impedance-matching problems. However, the problem lies in processing these materials with enough selectivity as compared to silicon oxide so that they are not etched away with the sacrificial oxide during the final release step. Anchor and electrode openings are then patterned and etched through the spacer oxide and the sacrificial oxide layers to provide direct electrical contact between the interconnects polysilicon layer and the anchor/electrode layer to be deposited next. Oxide spacer is etched away from the coupling-beam opening using a different mask. Following the etching of the sacrificial and spacer oxide to expose the coupling-beam opening (in structural disk) and interconnect layers, wafer is ash cleaned to remove any photoresist residuals. The third and last $2-\mu \mathrm{m}$ polysilicon layer forming anchor and electrodes are then deposited using LPCVD, doped using POCL doping, and capped with $0.5 \mu \mathrm{m}$ of hard-mask oxide deposited using LPCVD. The deposited polylayer refills the anchor openings and make both electrical and mechanical contacts to the interconnect polylayer. The pattern on the top oxide hard mask is then transferred to the underlying third poll area via RIE to form the input-output electrodes and anchors. Finally, the device is released using HF and dried using supercritical cleaning.

\section{CONCLUSION}

In this paper, a new design process for mechanically coupled vibrating RF MEMS filters was developed. The design process was applied to the wineglass-mode disk resonator to create HF (about $71 \mathrm{MHz}$ ) high- $Q$ filters with bandwidths of $1.14 \mathrm{MHz}$, $259 \mathrm{kHz}$, and $100 \mathrm{kHz}$. Such filters can find their way to replace bulky and off-chip crystal and SAW high- $Q$ bandpass filters, commonly used in the RF and IF stages of heterodyning transceivers. By doing so, it should be feasible to develop

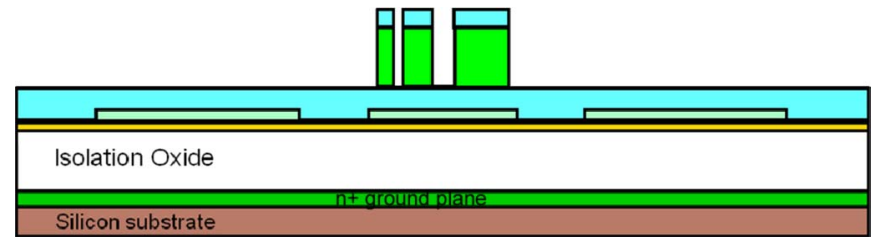

Fig. 8. Second polysilicon layer is patterned to define central stem anchor and overhanging coupling-beam opening.

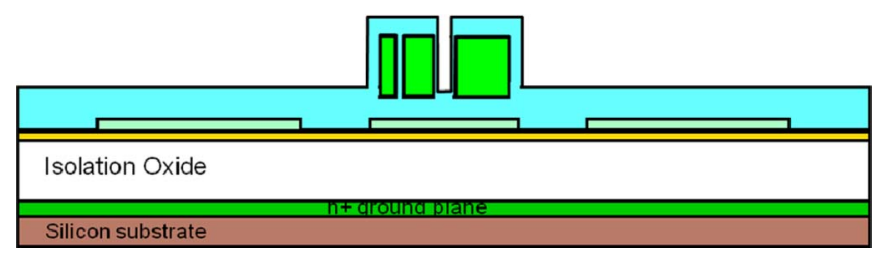

Fig. 9. Oxide spacer is deposited to define the side air gap.

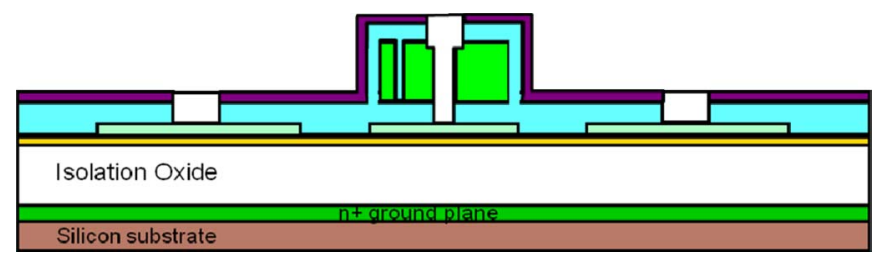

Fig. 10. Photoresist is patterned to open contacts through sacrificial oxide to the underlying poly1 interconnect layer.

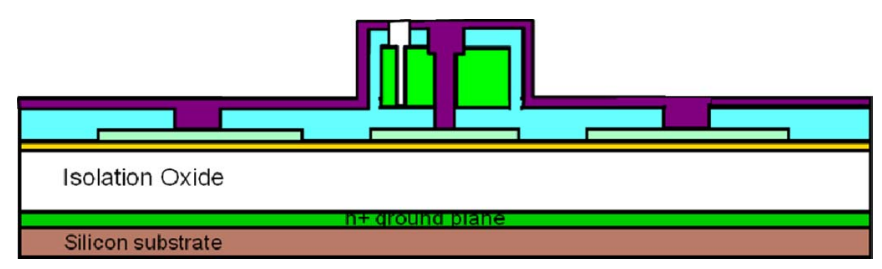

Fig. 11. Photoresist is patterned to etch the gap oxide from the coupling-beam opening.

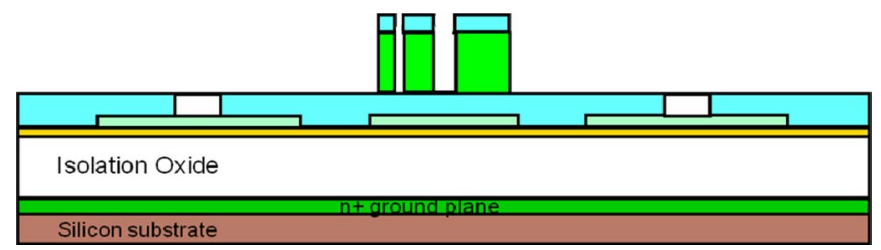

Fig. 12. Wafer is ashed to remove any photoresist residuals before depositing the third polysilicon layer.

low-cost single-chip wireless transceiver that can be used in an autonomous sensors deployed in WSNs for an array of applications.

Simulated annealing was used to find the coupling location and the coupling-beam dimensions to obtain a desired filter bandwidth. The algorithm was implemented to design a filter with bandwidth of $100 \mathrm{kHz}$ as an example. The algorithm can also be implemented to any other desired bandwidth.

Upon the experimentation of the equations governing the bandwidth, it can be deduced that the design is mostly sensitive to slight tolerances in the beamwidth. This is because the 


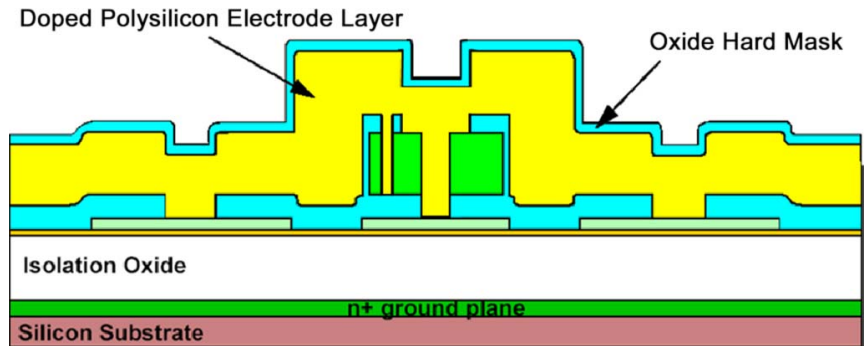

Fig. 13. Third polysilicon layer (defining in/out electrodes and coupling beam) is deposited, doped, and capped with oxide hard mask.

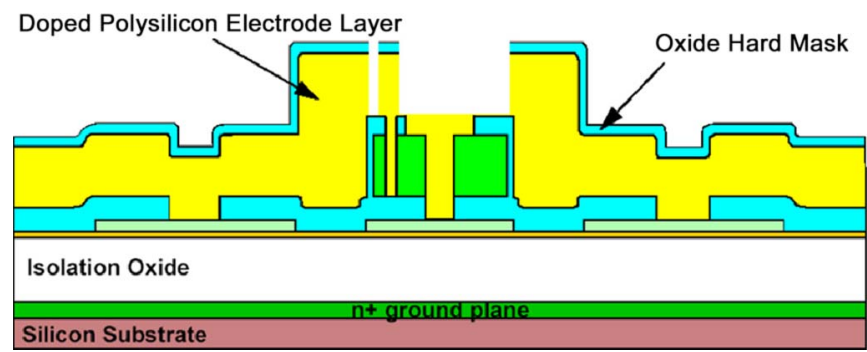

Fig. 14. Third polysilicon layer is patterned to define in/out electrodes and overhanging coupling beam.

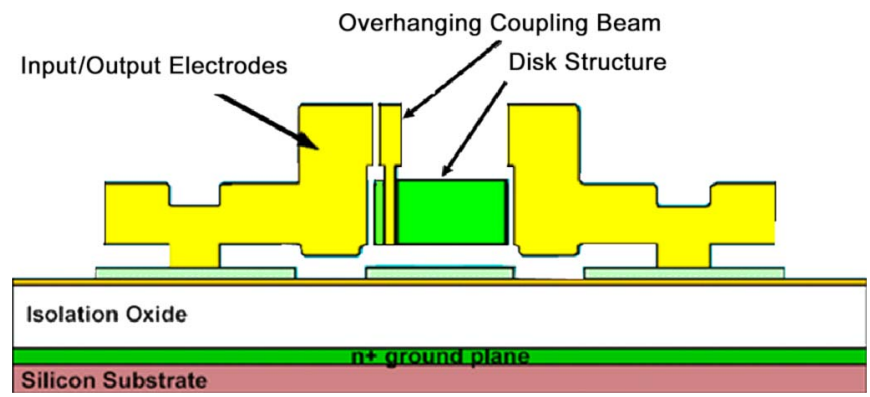

Fig. 15. Wet etch of sacrificial oxide and gap spacing oxide releasing the filter structure.

current fabrication techniques have no accurate control on such dimension. The variation in the beamwidth can be around $0.2 \mu \mathrm{m}$. With a width of $0.82 \mu \mathrm{m}$, the tolerance is $24 \%$. This means that at least the bandwidth will also vary by $24 \%$. In addition, a variation in the beamwidth will violate (9) for flexural-mode beam coupling, and so, the filter center frequency may be affected. Since there are no analytical equations model, the effect of the coupling-beam mass on the filter center frequency, tedious finite-element simulations would be needed to do complete sensitivity analysis for the designed filter.

Finally, the fabrication process is similar to regular MUMPS process. The only slight variation is that it needs the creation a nanogap between the electrodes and the resonator disks. This is done by depositing a very thin sacrificial oxide layer (OX2) of $0.1-\mu \mathrm{m}$ thickness.

As a future work, the authors plan to fabricate and test the optimized filter to verify the obtained simulation results.

\section{ACKNOWLEDGMENT}

This paper was prepared as part of a MEMS course taught by Prof. K. Kurabayashi, Department of Mechanical Engineering, University of Michigan.

\section{REFERENCES}

[1] A. Flammini, P. Ferrari, D. Marioli, E. Sisinni, and A. Taroni, "Sensor networks for industrial applications," in Proc. 2nd IEEE Int. Workshop Advances Sens. Interfaces, 2007, pp. 76-90.

[2] B. Lu, T. G. Habetler, R. G. Harley, and J. A. Gutierrez, "Applying wireless sensor networks in industrial plant energy management systems-Part I: A closed-loop scheme," in Proc. IEEE Sens., 2005, pp. $145-150$.

[3] J. M. Quero, C. Aracil, L. G. Franquelo, R. Ricart, P. R. Ortega, M. Domínguez, L. M. Castañer, and R. Osuna, "Tracking control system using an incident radiation angle microsensor," IEEE Trans. Ind. Electron., vol. 54, no. 2, pp. 1207-1216, Apr. 2007.

[4] M. Swaminathan, A. Bavisi, W. Yun, V. Sundaram, V. Govind, and P. Monajemi, "Design and fabrication of integrated RF modules in liquid crystalline polymer (LCP) substrates," in Proc. IEEE IECON, Nov. 6-10, 2005, pp. 2346-2351.

[5] M. Maddela, R. Ramadoss, and R. Lempkowski, "PCB MEMSbased tunable coplanar patch antenna," in Proc. ISIE, Jun. 4-7, 2007, pp. 3255-3260.

[6] R. G. Bolea, A. Luque, and J. M. Quero, "Capacitive pressure sensor and characterization as RF MEMS devices," in Proc. IEEE ISIE, Jun. 4-7, 2007, pp. 3267-3272.

[7] A. Elzayat, M. Degbe, F. Domingue, and V. Nerguizian, "A suspended MEMS inductor of a new shape and a novel anchor for RF applications," in Proc. IEEE Int. Symp. Ind. Electron., Jul. 9-12, 2006, pp. 3332-3337.

[8] C. T.-C. Nguyen, "MEMS technology for timing and frequency control," IEEE Trans. Ultrason., Ferroelectr., Freq. Control, vol. 54, no. 2, pp. 251-270, Feb. 2007.

[9] D. Fries, H. Broadbent, G. Steimle, S. Ivanov, A. Cardenas-Valencia, J. Fu, M. Janowiak, T. Weller, L. Guerra, and S. Natarajan, "PCBMEMS for environmental sensing systems," in Proc. IEEE IECON, Nov. 6-10, 2005 , p. 5.

[10] C. T.-C. Nguyen, "Microelectromechanical components for miniaturized low-power communications," in Proc. IEEE MTT-S, Anaheim, CA, 1999, pp. $48-77$.

[11] C. T.-C. Nguyen, "Microelectromechanical circuits for communications transceivers," in Proc. BCTM, Minneapolis, MN, 2000, pp. 25-26.

[12] A. B. Carlson, Communication Systems, ser. Electrical and Electronic Engineering Series. New York: McGraw-Hill, 2001.

[13] B. Razavi, RF Microelectronics, ser. Prentice Hall Communications Engineering and Emerging Technologies Series. Englewood Cliffs, NJ: Prentice-Hall, 1997.

[14] R. G. Kinsman, Crystal Filters. Hoboken, NJ: Wiley, 1987.

[15] S. K. Mitra and C. F. Kurth, Miniaturized and Integrated Filters. Hoboken, NJ: Wiley, 1989.

[16] S. Datta, Surface Acoustic Wave Devices. Englewood Cliffs, NJ: Prentice-Hall, 1986.

[17] S. V. Krishnaswamy, J. R. Rosenbaum, S. S. Horwitz, and R. A. Moore, "Film bulk acoustic wave resonator and filter technology," in Tech. Dig. IEEE MTT-S, 1992, pp. 153-155.

[18] R. C. Ruby, P. Bradley, Y. Oshmyansky, and A. Chein, "Thin film bulk wave acoustic resonators (FBAR) for wireless applications," in Tech. Dig. IEEE Ultrason. Symp., 2001, pp. 813-821.

[19] P. Reddy and S. Ravi, "GA-tuned microelectromechanical filters for signal processing," in Proc. 7th ICSP, 2004, vol. 1, pp. 384-387.

[20] S.-S. Li, Y.-W. Lin, Z. Ren, and C. T. C. Nguyen, "Self-switching vibrating micromechanical filter bank," in Proc. IEEE Int. Freq. Control Symp. Expo., 2005, pp. 29-31.

[21] R. T. Howe and R. S. Muller, "Resonant microbridge vapor sensor," IEEE Trans. Electron Devices, vol. ED-33, no. 4, pp. 499-506, Apr. 1986.

[22] W. C. Tang, T.-C. H. Nguyen, and R. T. Howe, "Laterally driven polysilicon resonant microstructures," Sens. Actuators, vol. 20, pp. 25-32, 1989.

[23] R. Candler, W.-T. Park, H. Li, A. Partridge, M. Lutz, and T. Kenny, "Single wafer encapsulation of MEMS devices," IEEE Trans. Adv. Packag., vol. 26, no. 3, pp. 227-232, Aug. 2003.

[24] J. Teva, G. Abadal, A. Uranga, J. Verd, F. Torres, J. L. Lopez, J. Esteve, F. Pérez-Murano, and N. Barniol, "From VHF to UHF CMOS-MEMS monolithically integrated resonators," in Proc. IEEE Int. Conf. Micro Electro Mech. Syst., Tucson, AZ, Jan. 2008, pp. 82-85.

[25] W.-L. Huang, Z. Ren, Y.-W. Lin, H.-Y. Chen, J. Lahann, and C. T.-C. Nguyen, "Fully monolithic CMOS nickel micromechanical resonator oscillator," in Proc. IEEE Int. Conf. Micro Electro Mech. Syst., Tucson, AZ, Jan. 2008, pp. 10-13. 
[26] M. Koskenvuori, V. Kaajakari, T. Mattila, and I. Tittonen, "Temperature measurement and compensation based on two vibrating modes of bulk acoustic mode microresonator," in Proc. IEEE Int. Conf. Micro Electro Mech. Syst., Tucson, AZ, Jan. 2008, pp. 78-81.

[27] M. Abdelmoneum, M. Demirci, and C. Nguyen, "Stemless wineglass-mode disk micromechanical resonators," in Proc. IEEE 16th Annu. Int. Conf. Micro Electro Mech. Syst., Kyoto, Japan, Jan. 19-23, 2003, pp. 698-701.

[28] C. Nguyen and M. Abdelmoneum, "Stemless wine glass mode disk resonators," International Patent H03H009/24, Jan. 10, 2006. U. S. Patent Office 333199000.

[29] Y.-W. Lin, S. Lee, S.-S. Li, Y. Xie, Z. Ren, and C. Nguyen, "Seriesresonant VHF micromechanical resonator reference oscillators," IEEE J. Solid State Circuits, vol. 39, no. 12, pp. 2477-2491, Dec. 2004.

[30] K. Wang, F. Bannon, J. Clark, and C. Nguyen, "Q-enhancement of microelectromechanical filters via low velocity spring coupling," in Proc. IEEE Int. Ultrason. Symp., Toronto, ON, Canada, Oct. 5-8, 1997, pp. 323-327.

[31] F. Bannon, J. Clark, and C. Nguyen, "High-Q HF microelectromechanical filters," IEEE J. Solid-State Circuits, vol. 35, no. 4, pp. 512-526, Apr. 2000.

[32] D. Greywall and P. Busch, "Coupled micromechanical drumhead resonators with practical applications as electromechanical bandpass filters," J. Micromech. Microeng., vol. 12, no. 6, pp. 925-938, Nov. 2002.

[33] R. Johnson, Mechanical Filters in Electronics. New York: Wiley, 1987.

[34] M. Onoe, "Contour vibrations of isotropic circular plates," J. Acoust. Soc. Amer., vol. 28, no. 6, pp. 1158-1162, Nov. 1956.

[35] A. Zverev, Handbook of Filter Synthesis. New York: Wiley, 1967.

[36] K. Wang and C. Nguyen, "High-order micromechanical electronic filters," in Proc. 10th Annu. Int. Workshop Micro Electro Mech. Syst., Nagoya, Japan, Jan. 26-30, 1997, pp. 25-30.

[37] S. Kirkpatrick, C. D. Gellat, and M. P. Vecchi, "Optimization by simulated annealing," Science, vol. 220, no. 4598, pp. 671-680, May 1983.

[38] P. Laarhoven and E. Aarts, Simulated Annealing: Theory and Applications. Amsterdam, The Netherlands: Reidel, 1987.

[39] S. Szykman, L. Schmidt, and H. Shetty, "Improving the efficiency of simulated annealing optimization through detection of productive search," presented at the American Society Mechanical Engineers (ASME) Design Engineering Technical Conf. (DETC) Computers Information Engineering Conf., Sacramento, CA, Sep. 14-17, 1997, Paper DETC97/DAC3980.

[40] S. S. Rao, Engineering Optimization, Theory and Practice, 3rd ed. Hoboken, NJ: Wiley, 1999.

[41] M. Shalaby, "Topology optimization of structures using hybrid simulated annealing," M.S. thesis, Mech. Des. Prod. Dept., Cairo Univ., Giza, Egypt, 2002.

[42] J. Clark, W.-T. Hsu, M. Abdelmoneum, and C. T.-C. Nguyen, "High-Q UHF micromechanical radial-contour mode disk resonators," J. Microelectromech. Syst., vol. 14, no. 6, pp. 1298-1310, Dec. 2005.

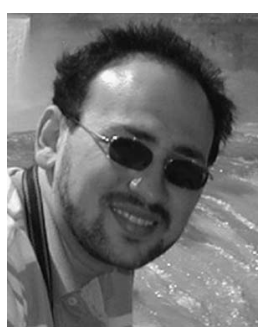

Mohammed M. Shalaby received the B.S. and M.S degrees in mechanical engineering from Cairo University, Cairo, Egypt, in 2000 and 2002, respectively, and the $\mathrm{Ph} . \mathrm{D}$. degree in mechanical engineering from the University of Michigan, Ann Arbor, in 2008.

Since February 2008, he has been with the General Electric Global Research Center, Niskayuna, NY, where he has been a Mechanical Engineer with the Mechanical Integration and Operability Laboratory. He has published over 20 conference/journal papers in the areas of design for disassembly, RF microelectromechanical systems (MEMS), and reverse engineering. His current research includes developing computer-aided-design tools for the automation of the design process, design automation and optimization, design for disassembly, and optimization of MEMS components.

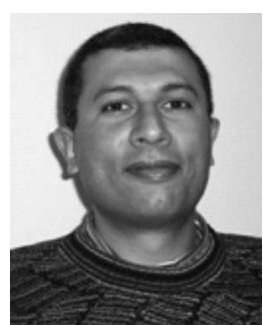

Mohamed A. Abdelmoneum (S'99-M'05) received the B.Sc. degree in electrical communications and electrophysics from Alexandria University, Alexandria, Egypt, in 1994, and the M.Sc. and Ph.D. degrees in electrical engineering and computer science from the University of Michigan, Ann Arbor, in 2000 and 2005, respectively.

Between January 1996 and May 1996, he was a Field Engineer with Schlumberger Wire Line and Testing. He was a Junior Faculty Member with the Department of Mathematics and Basic Sciences, Tanta University, Tanta, Egypt, where he worked on wavelets and their application to the solution of differential equations. In 1998, he was with the Radiation Laboratory, University of Michigan, where he worked on computational electromagnetics and its application to antenna design, radomes, frequency-selective surfaces, and electromagnetic computability. In 2001, he was with the Solid State Electronics Laboratory, where he worked on vibrating micromechanical resonators and filters. He focused on developing resonators capable of exhibiting high $Q$ 's under different environmental conditions as well as automatic postfabrication trimming of micromechanical resonators and filters. Since 2005, he has been with Intel Corporation, Hillsboro, OR, where he has been working on developing automated analysis systems for semiconductor process development for the 45- and 32-nm technologies and is currently with the Mobility Group working on developing microsensor technologies for wireless computing systems. He has published over 13 conference/journal papers in the areas of RF microelectromechanical systems (MEMS), computational electromagnetics, and semiconductor process development. He is the holder of three patents in the area of RF MEMS, with four pending.

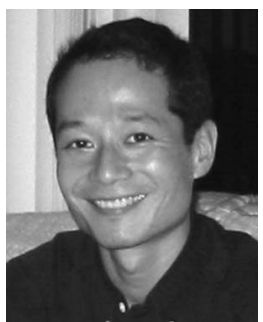

Kazuhiro Saitou (M'97-SM'07) received the B.S. degree in mechanical engineering from the University of Tokyo, Tokyo, Japan, in 1990, and the M.S. and $\mathrm{Ph} . \mathrm{D}$. degrees in mechanical engineering from Massachusetts Institute of Technology, Cambridge, in 1992 and 1996, respectively.

From 1997 to 2003, he was an Assistant Professor with the Department of Mechanical Engineering, University of Michigan, Ann Arbor, where he is currently an Associate Professor. His research interests include algorithmic and optimal synthesis of products and systems, including product/supply-chain codesign, optimal synthesis of microelectromechanical systems/NEMS, chemo/bioinformatics, and structure-based virtual screening in drug design.

Dr. Saitou is a member of the American Society of Mechanical Engineers (ASME) and Society of Manufacturing Engineers. He is currently serving as an Associate Editor for the IEEE TRANSACTIONS ON AUtOMATION SCIENCE AND ENGINEERING. He is currently the Vice Chair of the ASME Design for Manufacturing Technical Committee and a member of the ASME Design Automation Conference Executive Committee. He was the Organizing Chair of the 2007 IEEE International Symposium on Assembly and Manufacturing (ISAM07) and the Program Chair of the 2008 IEEE Conference on Automation Science and Engineering (IEEE CASE08) and 2008 ASME Design Automation Conference (DAC08). He was the recipient of a 1999 CAREER Award from the National Science Foundation, the Best Paper Award at the 5th International Symposium on Tools and Methods of Competitive Engineering in 2004, and the Outstanding Achievement Award from the Department of Mechanical Engineering at the University of Michigan in 2007. He was a finalist for the Best Paper Awards at EcoDesign: 4th International Symposium on Environmentally Conscious Design and Inverse Manufacturing in 2005 and the IEEE Conference on Automation Science and Engineering in 2006. 